\title{
Pattern of Intra Ocular Foreign Bodies in a Tertiary Care Hospital of Punjab
}

\author{
Usama Iqbal $^{1}$, Irfan Qayyum Malik ${ }^{2}$, Zeeshan Hameed ${ }^{3}$, Sadia Hameed ${ }^{4}$ \\ ${ }^{1-3}$ Department of Ophthalmology, Gujranwala Medical College, Gujranwala \\ ${ }^{4}$ Departmwent of Microbiology, Sheikh Zayed Hospital, Lahore
}

\section{Abstract}

Purpose: To find the epidemiological characteristics, management strategies and outcomes of intraocular foreign bodies (IOFB) in a tertiary care setup of Punjab.

Study Design: Descriptive retrospective study.

Place and Duration: Department of Ophthalmology, Gujranwala medical college/teaching hospital, from January 2017 to December 2019.

Methods: A retrospective review, of all the patients who had IOFB removal, was performed. Information regarding the nature and circumstances of injury, types of IOFB, operative procedure performed and patient's preoperative and post-operative best corrected visual acuity (BCVA) were analyzed. X-ray Orbit was advised to all the patients with ocular trauma having suspicion of any IOFB while CT scan was done in patients with negative Xray. Descriptive statistics were used for analysis of data.

Results: Record of 22 patients was retrieved out of which 18 (81.81\%) were male and 4 (18.18\%) were female with a mean age of $27.95 \pm 9.325$ years. Occupational trauma was the leading cause of injury $(66.6 \%)$. Metallic objects were among the most common type of IOFB (66.6\%) followed by glass, concrete stone (each $13.3 \%$ ) and lead pencil (6.6\%). Serious complications seen due to retained IOFB were phthisis bulbi $(9.09 \%)$, retinal detachment (13.63\%) and endophthalmitis (9.09\%). On follow-up, 10 patients had BCVA less than hand movement (HM), 5 patients had BCVA of CF to 6/60, 3 patients had BCVA of 6/60-6/24 and 4 patients had BCVA better than 6/18.

Conclusion: Occupational trauma in young age group of working class was the most common cause of IOFB. Treatment delay and complications contributed to poor visual prognosis.

Key Words: Intra-ocular foreign Body, Retinal Detachment, Phthisis Bulbi.

How to Cite this Article: Iqbal U, Malik IQ, Hameed Z, Hameed S. Pattern of Intra Ocular Foreign Bodies in a Tertiary Care Hospital of Punjab. Pak J Ophthalmol. 2021, 37 (1): 109-114.

Doi: https://doi.org/10.36351/pjo.v37i1.1071

\section{INTRODUCTION}

Intraocular Foreign Bodies (FB) are more commonly encountered in males in working age group. ${ }^{1}$ Injuries

Correspondence: Usama Iqbal

Department of Ophthalmology

Gujranwala Medical College, Gujranwala

Email: usamaiqqbal@gmail.com

Received: 2021

Accepted: 2021 to eye occur either by primary or secondary impact. Primary impact is direct mechanical injury while secondary impact is either introduction of infection in the eye or FB substance associated specific effects. ${ }^{1}$ Introduction of a FB in the eye can cause disturbing health issues ranging from transient ocular irritation to complete vision loss.

Ocular FBs can be classified in a number of ways. They are classified as extra ocular (EOFB) or intra ocular (IOFB) depending on whether FB is inside or outside the eyeball. ${ }^{2}$ This classification system seems 
simple. On the basis of exact location of FB, new classification systems evolved with time. These systems also include the terminology of adnexal foreign bodies (orbit, eyelids, conjunctiva, and lacrimal system) and intramural foreign bodies (IMFB) which are embedded within the layers of cornea or sclera and are neither IOFB nor EOFB. ${ }^{3}$

IOFBs are mainly chips of iron, copper, steel, glass, stones, lead and wood particles etc. The visual prognosis is dependent on multiple factors including age, extent of wound, time between injury and repair, size of FB, and complications such as, retinal detachment and endophthalmitis. ${ }^{4}$

A complete history and examination is usually required to determine the type and location of retained IOFB. Imaging plays a vital role in the diagnosis of IOFBs. Plain X-ray orbital radiography can be used for screening all the patients with penetrating eye injury. ${ }^{5}$ Additionally, Ultrasound and B-scan are also important modes of investigation, which can detect the size, location, nature of IOFB and complications caused by that FB. CT scan will detect small IOFBs and remains the investigation of choice when IOFB is not visible clinically. ${ }^{5}$ MRI is generally avoided especially if magnetic FB is suspected. ${ }^{6}$

Among penetrating ocular injuries, $18-40 \%$ present with retained IOFB..$^{7,8}$ In majority of cases, injury is mainly due to occupational trauma in adults and unsafe games in children. ${ }^{9}$ Keeping in view the modes of injury, majority of these injuries seem to be preventable and thus a number of people can be saved from getting blind through precautionary measures. ${ }^{9}$

In this article, we describe epidemiological characteristics and visual outcomes of patients with IOFB presenting to Ophthalmology Department of Gujranwala Medical College/Teaching Hospital during a period of 2 years. This study will be helpful in highlighting the importance of ocular safety measures.

\section{METHODS}

Our study area was Gujranwala city, which is situated $96.7 \mathrm{~km}$ north of the city of Lahore in Pakistan. Gujranwala is the $5^{\text {th }}$ most populous district of Pakistan. ${ }^{10}$ It is an industrial city with a large proportion of population working in industries and the who are at high risk for ocular trauma.

After permission from institutional review board, a retrospective case review study was conducted in which data of the patients from $1 \mathrm{st}^{\text {th }}$ January 2017 to $31^{\text {th }}$ December 2019 was analyzed. All patients who were operated in Ophthalmology Department for IOFB removal were included in the study. Ophthalmology Department Gujranwala Medical College/Teaching Hospital is the only ophthalmic unit in its locality providing 24/7 emergency cover to the patients of ocular trauma. Plain X-ray Orbit was advised to all the patients with ocular trauma having suspicion of any IOFB (Figure 1).

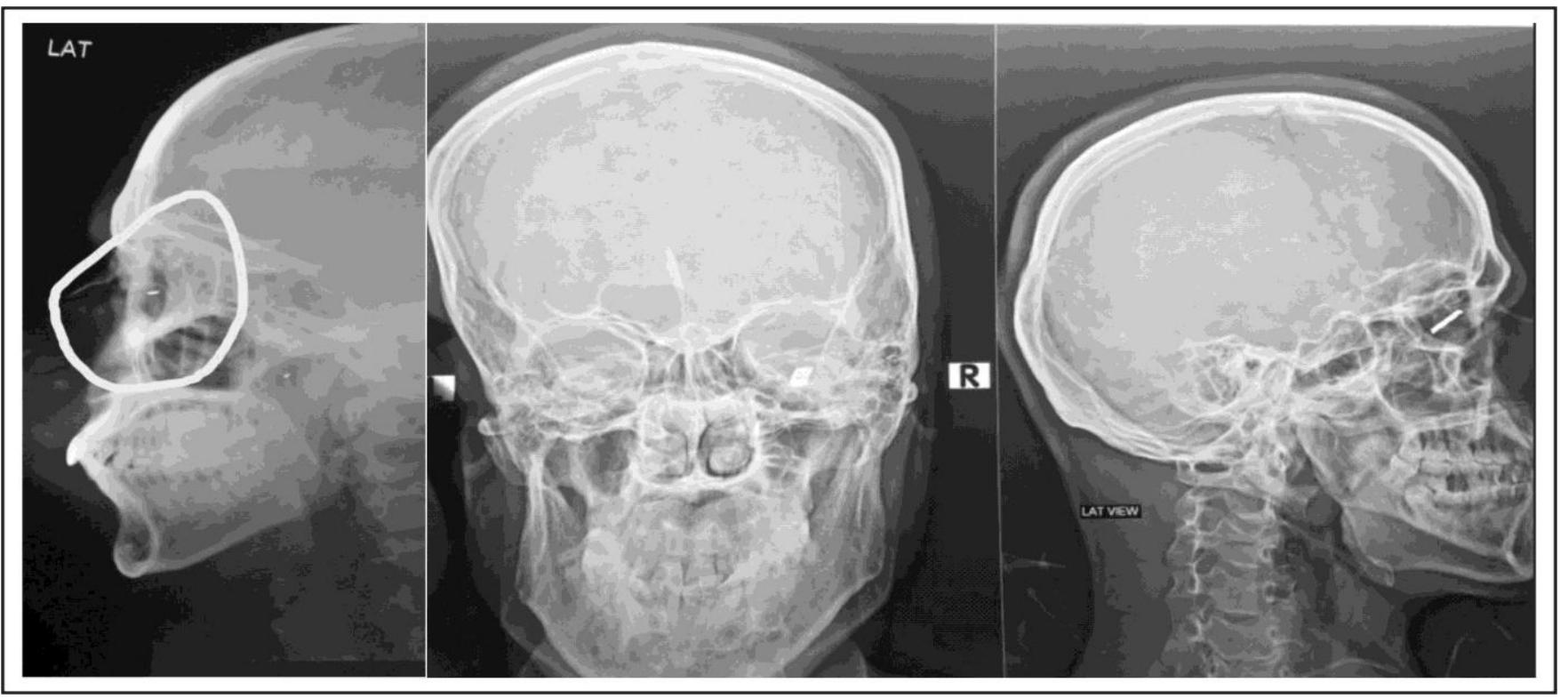

Fig. 1: Plain X-ray orbit showing IOFB. 


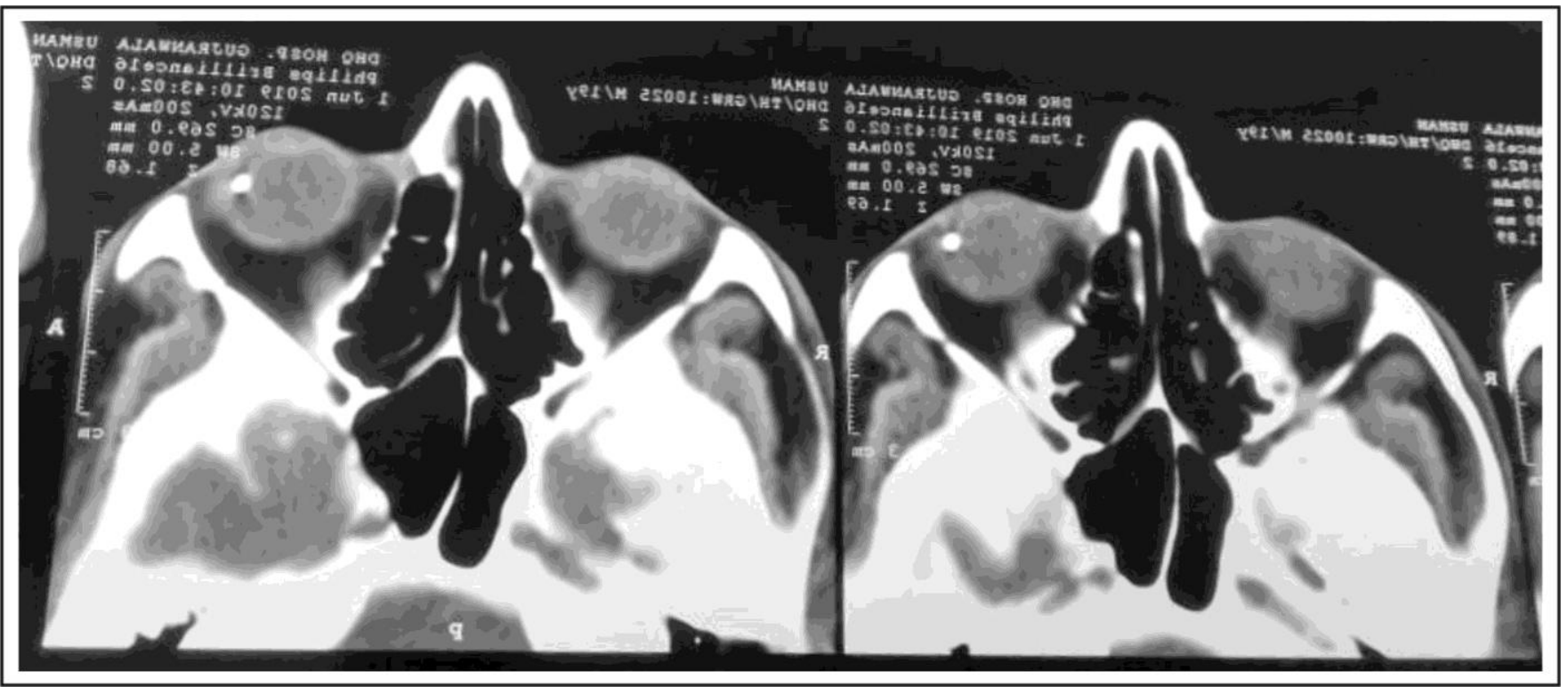

Fig. 2: CT scan orbit Axial View Showing R IOFB.

CT-Scan was advised in those cases, which involved negative findings on X-ray orbit but with clinical signs suggestive of Ocular FB (Figure 2).

Name, age, gender, contact details, occupation and clinical information like; nature and type of injury, type of tear, type of FB, other ocular findings, surgical procedure, pre and post-operative BCVA, duration of hospitalization, and impact of trauma on personal life were recorded.

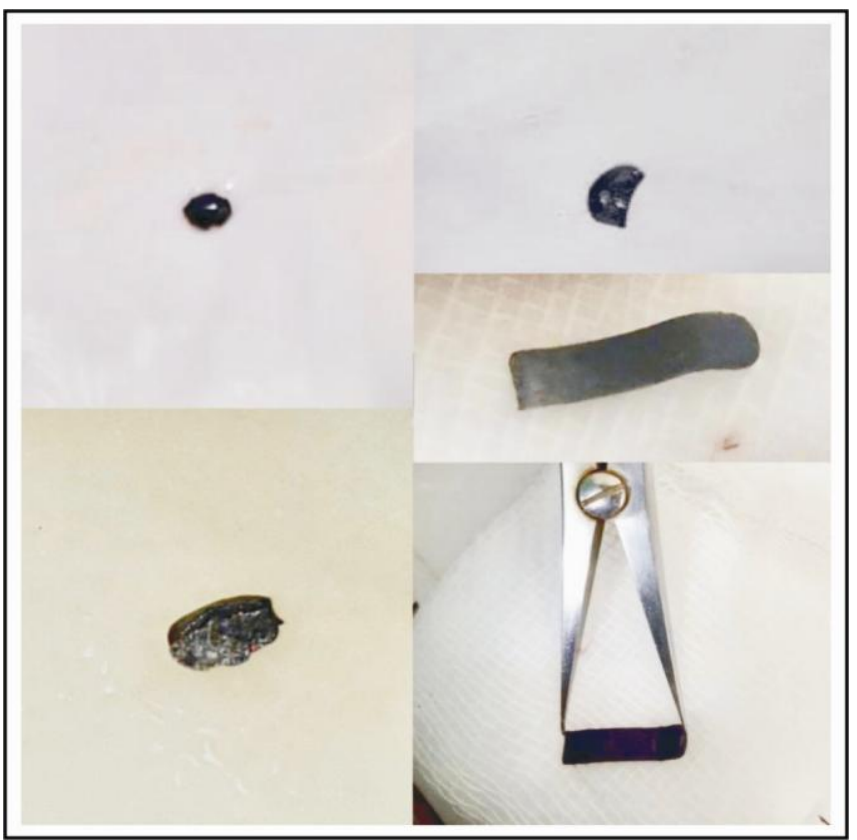

Fig. 3: Image showing different removed metallic IOFBs.

\section{RESULTS}

Total numbers of patients included in the study were 22. Mean age of the patients was $27.95 \pm 9.325$ years. Patients were categorized into three age groups (Table 1).

Table 1: Frequency Distribution of Gender and Age.

\begin{tabular}{lcc}
\hline & $\begin{array}{c}\text { Frequency } \\
(\mathbf{n = 2 2})\end{array}$ & Percentage \\
\hline Gender & 18 & \\
Male & 04 & $81.81 \%$ \\
Female & 22 & $18.18 \%$ \\
Total & & $100.0 \%$ \\
Age Groups & 3 & \\
1-18 years & 15 & $13.63 \%$ \\
18-35 years & 4 & $68.18 \%$ \\
$>$ 35 years & 22 & $18.18 \%$ \\
Total & & $100.0 \%$ \\
\hline
\end{tabular}

On the basis of location 14 patients $(63.63 \%)$ had IOFB in posterior segment while 8 patients $(36.36 \%)$ had IOFB in anterior segment (Figures $4 \& 5$ ).

Magnetic metallic objects were the commonest type of IOFB (66.6\%), followed by Glass, Concrete stone (each $13.3 \%$ ) and lead pencil (6.6\%). One aspect of injury was the involvement of visual axis. In 14 patients $(63.63 \%)$ visual axis was involved while in remaining 8 patients $(36.36 \%)$ there was no involvement of visual axis. 


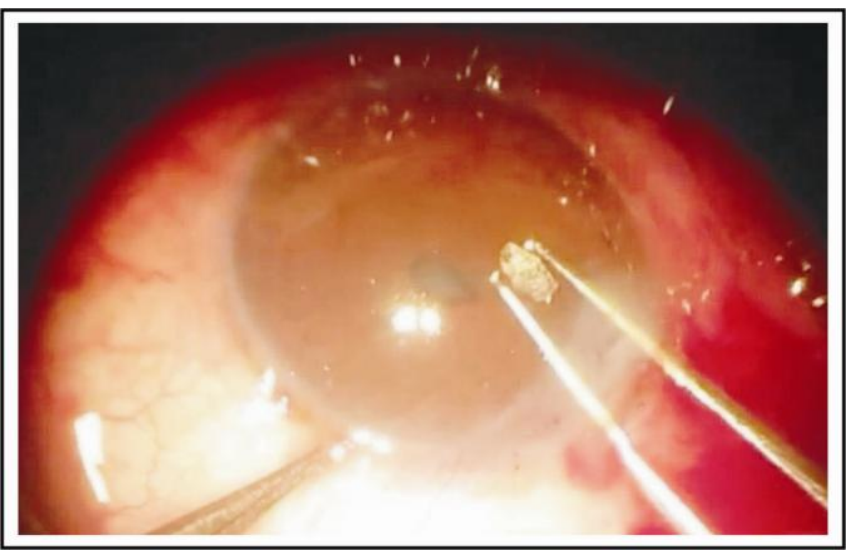

Fig. 4: IOFB removed from Anterior Chamber (AC) with the help of forceps.

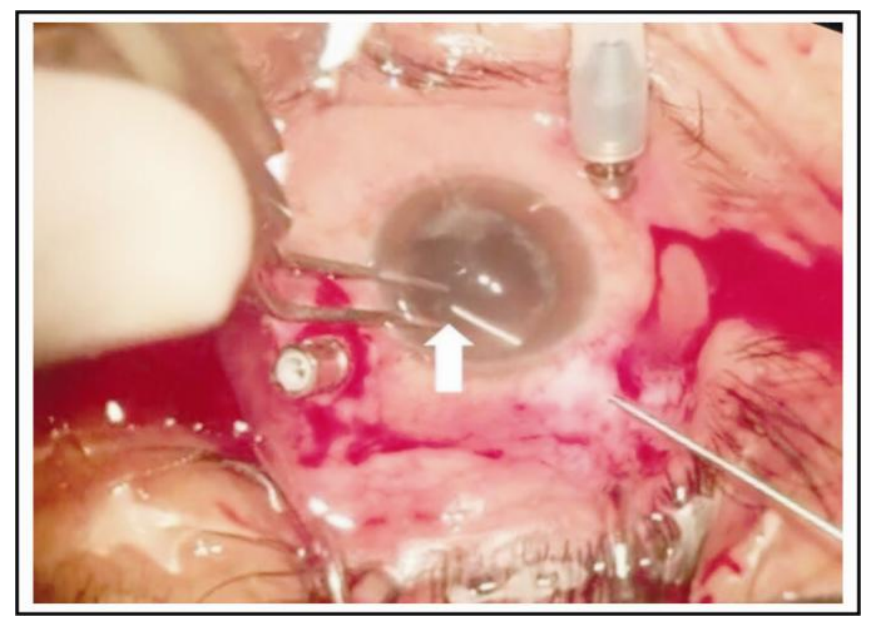

Fig. 5: Posterior segment IOFB engaged with the help of endomagnet and removed through corneal incision with the help of forceps.

Corneal entry wound was seen in 10 individuals (46.45\%), scleral entry in 6 patients $(27.27 \%)$ and corneo-scleral entry in 6 individuals $(27.27 \%)$. Left eye was involved in 13 patients $(60 \%)$ and right eye in 9 patients $(40 \%)$. Causes of trauma are shown in table 2.

Table 2: Frequency Distribution of Cause of Injury.

\begin{tabular}{lcc}
\hline Cause of Injury & Frequency & Percentage \\
\hline Occupational Trauma & 13 & 59.09 \\
Accidents & 6 & 27.27 \\
Others & 3 & 13.63 \\
Total $(\mathrm{n}=15)$ & 22 & 100.0 \\
\hline
\end{tabular}

Gap between time of presentation and surgical intervention greatly affects the visual prognosis. For details refer to table 3 .
Table 3: Frequency Distribution of Patient's Time of Presentation.

\begin{tabular}{lcc}
\hline Time of Presentation & Frequency & Percentage \\
\hline Within 1 hour & 3 & 13.63 \\
1- 24 hours & 6 & 27.27 \\
24- 48 hours & 5 & 22.72 \\
2 days - 1 week & 7 & 31.81 \\
1 week - 1 month & 1 & 4.5 \\
Total & 22 & 100.0 \\
\hline
\end{tabular}

IOFB was successfully removed in all patients. 23guage pars plana vitrectomy was performed in 14 patients. In 12 patients, IOFB was removed with the help of endo-magnet, 2 patients had non-magnetic or large sized IOFB which was grabbed with vitrectomy forceps and then removed through anterior segment. Silicon oil was injected in all the 14 patients who had IOFB removed from posterior segment. Endo-laser was applied in all the patients who had retinal break per-operatively. Most common location of retinal break was at 6'o clock position. Anterior segment IOFBs were removed through the corneal incision.

Corneal tear repair was performed in 8 patients, corneo-scleral tear repair in 6 patients and scleral tear repair in 6 patients. Two patients had self-healing corneal entry wounds. Traumatic Cataract was removed in 5 patients. Serious complications of IOFB were Phthisis Bulbi in 2 patients $(9.09 \%)$, retinal detachment in 3 patients $(13.63 \%)$ and endophthalmitis in 2 patients $(9.09 \%)$.

The main criterion of prognostic measure in follow-up was Best Corrected Visual Acuity (BCVA). For details see table 4 .

Table 4: Frequency Distribution of BCVA at Follow-up.

\begin{tabular}{lcr}
\hline Patient's BCVA & $\begin{array}{c}\text { Frequency } \\
(\mathbf{n = 2 2})\end{array}$ & Percentage \\
\hline No perception of light (NPL) & 2 & $9.09 \%$ \\
Perception of light & 3 & $13.63 \%$ \\
Hand Movements & 5 & $22.72 \%$ \\
CF-6/60 & 5 & $22.72 \%$ \\
6/60-6/24 & 3 & $13.63 \%$ \\
6/18 to 6/9 & 4 & $18.18 \%$ \\
\hline
\end{tabular}

\section{DISCUSSION}

Our study comprised of a total of 22 patients. Most of the studies published for epidemiological characteristics and management outcomes of IOFBs are retrospective. ${ }^{6,11,12}$ In our study design, data was also collected in retrospective manner. Majority of 
injuries $(81.81 \%)$ occurred in male group. Mean age of presentation was 27 years. Liu et al described similar trends with male predominance but mean age was 38 years. ${ }^{4}$ In another study, done for IOFBs in New York, male predominance with mean age of above 30 years was reported. ${ }^{13}$ Local authors have also reported mean age above 30 years. ${ }^{6,14}$ Young age group in our study might be because of small sample size. It can also be because of the reason that the study area was an industrial city with most of the laborers belonging to the age group of 20 to 30 years.

Regarding the type of IOFB, magnetic metals are the most common reported in literature which is also depicted in our study. ${ }^{15,16} \mathrm{~A}$ retrospective analysis of 1340 cases of IOFBs over 10 years period, reported farming as the most common profession. ${ }^{11}$ In other reports industrial workers were the most common patients presenting with IOFB. ${ }^{4,16}$ Hammer and chisel is also reported as the leading cause by some authors. ${ }^{15}$ In our study occupational trauma related to ceramic and utensil making industry, was the leading cause; followed by accidental injuries which included hammering related trauma to eye. The cause can vary from region to region. It depends on the type of study area and its population. None of the patients presented to us were using eye safety googles at the time of trauma. Most common point of entry was cornea. Overall corneal involvement was seen in $73.72 \%$ cases. Other local authors have also reported cornea as the most common entry point for IOFB. ${ }^{15,17}$ Some authors reported corneal involvement as high as $88 \% .^{13}$

In our study all the patients with clinical signs suggestive of IOFB were advised plain orbital X-ray. The sensitivity of plain orbital X-rays for radio-opaque foreign bodies ranges from 70-90\% and CT-scan remains the gold standard investigation for IOFB..$^{5}$ In our study only one patient was diagnosed with IOFB on CT-scan with no finding on plain X-ray.

Time of presentation to emergency ophthalmic unit and timely intervention is the most important factor, which can affect the visual prognosis. Only $13 \%$ patients presented within the first one hour. Idris et al., reported $27 \%$ patients presenting to eye care facility after ocular trauma. ${ }^{6}$

The type of instrument to be used for removal of posterior segment IOFB depends on the location, size and nature of IOFB. ${ }^{18}$ Poor presenting visual acuity is regarded as the most important factor which determines the final visual outcome. ${ }^{19,20}$ Other factors which influence final VA include the size of IOFB, age of patient and associated complications (retinal break, endophthalmitus, retinal detachment). ${ }^{4}$ Decision regarding removal of difficult IOFB keeping in mind the risk of surgery can vary from case to case. With improvement in surgical techniques and surgical devises, the decision to plan for removal of IOFB is now easier. Some authors recommend removal of IOFB in all cases regardless of its location and inertness. $^{21}$

Limitations of our study include its retrospective design and small sample size. Information regarding instruments and exact surgical technique used for removal of IOFB were also not available.

\section{CONCLUSION}

Ocular trauma leading to intra ocular foreign bodies is one of the major challenges faced by an Eye surgeon. Since occupational trauma is the leading cause of such injuries especially in young age group, so proper care during working hours can reduce the risk of trauma to a great extent.

Ocular safety measures and protective equipment should be provided to all the industrial workers who are at high risk of ocular trauma and its related complications. The licensing agencies regulating these industries should also ensure the availability of safety equipment for the workers.

\section{Ethical Approval}

The study was approved by the Institutional review board/ Ethical review board. (Admn.112/GMC)

\section{Conflict of Interest}

Authors declared no conflict of interest.

\section{REFERENCES}

1. Pandey AN. Ocular Foreign Bodies: A Review. J Clin Exp Ophthalmol. 2017; 8: 1-5.

2. Duke-Elder S, MacFaul PA. System of Ophthalmology. Part I. 1st ed. London: Henry Kimpton; 1972: e451-501.

3. Shukla B. New classification of ocular foreign bodies. Chin J Traumatol. 2016; 19 (6): 319-321.

4. Liu Y, Wang S, Li Y, Gong Q, Su G, Zhao J. Intraocular foreign bodies: Clinical characteristics and prognostic factors influencing visual outcome and globe survival in 373 eyes. J Ophthalmol. 2019; 2019. 
5. Saeed A, Cassidy L, Malone DE, Beatty S. Plain Xray and computed tomography of the orbit in cases and suspected cases of intraocular foreign body. Eye, 2008; 22 (11): 1373-1377.

6. Idris M, Ayaz S, Yaqoob H. Demographic Characteristics of Cases with IOFB Presented to a Tertiary Care Centre. Pak J Ophthalmol. 2015; 31 (1): 22-26.

7. Loporchio D, Mukkamala L, Gorukanti K, Zarbin M, Langer P, Bhagat $\mathbf{N}$. Intraocular foreign bodies: a review. Surv Ophthalmol. 2016; 61 (5): 582-596.

8. Han S, Wang T, Jia J, Sun S, Fan Y, Yang G, et al. Visual outcomes and prognostic factors of intralenticular foreign bodies in a tertiary hospital in North China. J Ophthalmol. 2019; 2019: 4964595. doi.org/10.1155/2019/4964595

9. Romaniuk VM. Ocular trauma and other catastrophes. Emerg Med Clin N Am. 2013; 31 (2): 399-411.

Doi: 10.1016/j.emc.2013.02.003.

10. Iqbal U, Malik IQ, Iqbal H. Epidemiology of Ocular Trauma in a Tertiary Hospital Setting. Pak J Ophthalmol. 2019; 35 (1): 47-54.

11. Li L, Lu H, Ma K, Li YY, Wang HY, Liu NP. Etiologic causes and epidemiological characteristics of patients with intraocular foreign bodies: retrospective analysis of 1340 cases over ten years. J Ophthalmol. 2018; 2018: 6309638.

12. Luo $\mathbf{Z}$, Gardiner $\mathbf{M}$. The incidence of intraocular foreign bodies and other intraocular findings in patients with corneal metal foreign bodies. Ophthalmology, 2010; 117 (11): 2218-2221.

13. Chae B, Cohen EJ, Cymerman RM, Park L. Epidemiology, clinical characteristics and complications in ocular foreign body injuries. Invest Ophthalmol Vis Sci. 2014; 55 (13): 4713.

14. Ademola IW, Naha N, Boladale IA. Clinical and Demographic Characteristics of Intraocular Foreign Body Injury in a Referral Center: 3 Years' Experience. Pak J Ophthalmol. 2016; 32 (4): 205-209.

15. Khan N, Waheed K, Siddiq S, Tayyib M. Visual outcome and complications in intra ocular foreign bodies. Pak Armed Forces Med J. 2014; 64 (4): 509513.
16. Napora KJ, Obuchowska I, Sidorowicz A, Mariak Z. Intraocular and intraorbital foreign bodies characteristics in patients with penetrating ocular injury. Klinika Oczna. 2009; 111 (10-12): 307-312.

17. Memon AA, Iqbal MS, Cheema A, Niazi JH. Visual outcome and complications after removal of posterior segment intraocular foreign bodies through pars plana approach. J Coll Physicians Surg Pak. 2009; 19 (7): 436-439.

18. Jahangir T, Qureshi BZ, Chaudhry QL, Khan AA. Ease of Removal of Posterior Segment Metallic Intraocular Foreign Body with Intraocular Forceps Vs Endomagnet Plus Forceps. Pak J Ophthalmol. 2014; 30 (2): 95-98.

19. Valmaggia C, Baty F, Lang C, Helbig H. Ocular injuries with a metallic foreign body in the posterior segment as a result of hammering: the visual outcome and prognostic factors. Retina. 2014; 34 (6): 111611122.

20. Ma J, Wang Y, Zhang L, Chen M, Ai J, Fang X. Clinical characteristics and prognostic factors of posterior segment intraocular foreign body in a tertiary hospital. BMC ophthalmology, 2019; 19 (1): 1-6.

21. Jastaneiah SS. Long-term corneal complication of retained anterior chamber-angle foreign body. Saudi J Ophthalmol. 2010; 24 (3): 105-108.

\section{Authors' Designation and Contribution}

Usama Iqbal; Post Graduate Resident: Permission from institutional review board, Manuscript writing, final review and correspondence.

Irfan Qayyum Malik; Associate Professor: Supervised the whole project, formulated the study design and performed surgeries of posterior segment IOFB presented in this study, review the final manuscript.

Zeeshan Hameed; House officer: Data collection, Data entry and manuscript writing.

Sadia Hameed; Post Graduate Resident: Final review, Statistical analysis and Data handling. 